\title{
Approximations and Applications of a Reciprocal Fifth Power Mapping
}

\author{
S. Merlin, B. V. Senthil Kumar
}

\begin{abstract}
The approximation of different rational form of equations involving functions on both sides is an interesting study in the research topic of classical approximation of equations. The intention of this study is to obtain approximate reciprocal fifth power mapping through classical stability theory and to link the equations dealt in this study with various postulations occurring in physics, chemistry and mechanics.
\end{abstract}

Keywords : Non-Archimedean field, Quintic functional equation, Reciprocal functional equation, Ulam-Hyers stability.

\section{INTRODUCTION}

The fundamental question and various developments of theory of functional equations are available in the literature in ([5], [6], [8], [9], [10], [16]). There are many novel inspiring, interesting and significant results associated with stabilities of different types of equations, for details, one can refer to [1], [2], [3], [7], [11], [12], [13], [14], [15].

In this paper, a new reciprocal fifth power mapping satisfying the ensuing equation

$$
m_{f}\left(r_{1}+r_{2}\right)=\frac{m_{f}\left(r_{1}\right) \mathrm{m}_{f}\left(r_{2}\right)}{\left(m_{f}\left(r_{1}\right)^{\frac{1}{5}}+m_{f}\left(r_{2}\right)^{\frac{1}{5}}\right)^{5}}
$$

is introduced. Then its classical stability results are investigated in the setting of fields without non-Archimedean property. A suitable counter-example is presented to invalidate the stability result for a critical case. It is easy to verify that the reciprocal fifth power mapping $m_{f}(r)=r^{-5}$ is a solution of (1). Hence, (1) is called as reciprocal fifth power functional equation. The solution of (1) is linked with various hypotheses arising in physics, chemistry and mechanics.

\section{PRELIMINARIES}

Here, we reminisce about few basic definitions relevant

Definition 1: Suppose $K$ is a field. Let a function $|\cdot|: K \rightarrow[0, \infty)$ be defined on $K$. Then $K$ is said to be a non-Archimedean field, if the ensuing axioms are fulfilled: (i) $|\gamma|=0$,

Revised Manuscript Received on December 15, 2019.

S. Merlin, Assistant Professor, Department of Mathematics, Kalasalingam Academy of Research and Education, Anand Nagar, Tamilnadu, India.

Dr. B. V. Senthil Kumar, Department of Information Technology, Nizwa College of Technology, Nizwa, Oman. to non-Archimedean fields.

(ii) $|\gamma+\delta|=|\gamma \| \delta|$ and

(iii) $|\gamma+\delta| \leq \max \{|\gamma|,|\delta|\}$, for all $\gamma, \delta \in K$. Then, it is obvious that $|1|=|-1|=1$ and $|n| \leq 1$ for all non-negative integers $n$. Further, we presume that, $|\cdot|$ is not trivial, that is, there exists a $\lambda_{0} \in K$ such that $\lambda_{0} \neq 0,1$. In lieu, of the inequality: $\left|r_{y}-r_{x}\right| \leq \max \left\{\left|r_{k+1}-r_{k}\right|: x \leq k \leq y-1\right\}(y>x)$, a sequence $\left\{r_{x}\right\}$ becomes Cauchy if and only if $\left\{r_{x+1}-r_{x}\right\}$ approaches zero in a non-Archimedean field. If every Cauchy sequence converges in non-Archimedean field, then it is said to be complete.

\section{APPROXIMATIONS OF RECIPROCAL FIFTH POWER MAPPING}

In the entire investigation, let us assume that $S$ to be a field without non-Archimedean property and $T$ be a complete field without non-Archimedean property. In the upcoming outcomes, let us indicate $S^{*}=S \backslash\{0\}$. Let $R$ denote the space of real numbers.

Definition 2. If a mapping $m_{f}: S^{*} \rightarrow T$ satisfies (1), then it is called as reciprocal fifth power mapping.

For the simplification purpose in proving the main results, let us delineate the difference operator

$$
\Delta m_{f}\left(r_{1}, r_{2}\right)=m_{f}\left(r_{1}+r_{2}\right)-\frac{m_{f}\left(r_{1}\right) m_{f}\left(r_{2}\right)}{\left(m_{f}\left(r_{1}\right)^{\frac{1}{5}}+m_{f}\left(r_{2}\right)^{\frac{1}{5}}\right)^{5}}
$$

for all $r_{1}, r_{2} \in S^{*}$. In this section, we approximate reciprocal fifth power mapping via investigating various fundamental stabilities associated with (1) in non-Archimedean fields.

Theorem 1. Let $\lambda= \pm 1$ be fixed, and let $\Psi: S^{*} \times S^{*} \rightarrow \infty$ be a mapping such that

$$
\lim _{k \rightarrow \infty}\left|\frac{1}{32}\right|^{\lambda k l} \Psi\left(\frac{r_{1}}{2^{\lambda k+\frac{\lambda+1}{2}}}, \frac{r_{2}}{2^{\lambda k+\frac{\lambda+1}{2}}}\right)=0
$$

for all $r_{1}, r_{2} \in S^{*}$. Suppose that $m_{f}: S^{*} \rightarrow T$ is a mapping satisfying the inequality

$$
\left|\Delta m_{f}\left(r_{1}, r_{2}\right)\right| \leq \Psi\left(r_{1}, r_{2}\right)
$$

for all $r_{1}, r_{2} \in S^{*}$. Then, a unique reciprocal fifth power mapping $M_{f}: S^{*} \rightarrow T$ exists such that

$\left|m_{f}(r)-M_{f}(r)\right|$

$\leq \sup \left\{\left|\frac{1}{32}\right|^{\lambda \alpha+\frac{\lambda-1}{2}} \Psi\left(\frac{r}{2^{\lambda \alpha+\frac{\lambda+1}{2}}}, \frac{r}{2^{\lambda \alpha+\frac{\lambda+1}{2}}}\right): \alpha \geq 0\right.$ is an integer $\}$ 


\section{Approximations and Applications of a Reciprocal Fifth Power Mapping}

for all $u \in S^{*}$.

Proof. Let us instigate $\left(r_{1}, r_{2}\right)$ by $\left(\frac{r}{2}, \frac{r}{2}\right)$ in (3) to get

$$
\left|m_{f}(\mathrm{r})-\frac{1}{32^{\lambda}} m_{f}\left(\frac{r}{2^{\lambda}}\right)\right| \leq|32|^{\frac{|\lambda-1|}{2}} \Psi\left(\frac{r}{2^{\frac{\lambda+1}{2}}}, \frac{r}{2^{\frac{\lambda+1}{2}}}\right)
$$

for all $r \in S^{*}$. Then, now replacing $r$ by $\frac{r}{2^{\lambda k}}$ in (5) and multiplying by $\left|\frac{1}{32}\right|^{\lambda k}$, we have

$$
\begin{aligned}
\left|\frac{1}{32^{\lambda k}} m_{f}\left(\frac{r}{2^{\lambda k}}\right)-\frac{1}{32^{(k+1) \lambda}} m_{f}\left(\frac{r}{2^{(k+1) \lambda}}\right)\right| \\
\leq\left|\frac{1}{2}\right|^{m \beta+\frac{\beta-1}{2}} \xi\left(\frac{u}{2^{m \beta+\frac{\beta+1}{2}}}, \frac{u}{2^{m \beta+\frac{\beta+1}{2}}}\right)
\end{aligned}
$$

for all $r \in S^{*}$. Using the inequalities (2) and (6), we find that the sequence $\left\{\frac{1}{32^{\lambda k}} m_{f}\left(\frac{r}{2^{\lambda k}}\right)\right\}$ is Cauchy. By the completeness of $T$, this sequence converges to a mapping $M_{f}: S^{*} \rightarrow T$ defined by

$$
M_{f}(r)=\lim _{k \rightarrow \infty} \frac{1}{32^{\lambda k}} m_{f}\left(\frac{r}{2^{\lambda k}}\right) .
$$

Further, for every $r \in S^{*}$ and integers $k \geq 0$, we have

$$
\begin{aligned}
& \left|\frac{1}{32^{\lambda k}} m_{f}\left(\frac{r}{2^{\lambda k}}\right)-m_{f}(r)\right| \\
& \quad=\left|\sum_{j=0}^{k-1}\left\{\frac{1}{32^{(j+1) \lambda}} m_{f}\left(\frac{r}{2^{(j+1) \lambda}}\right)-\frac{1}{32^{j \lambda}} m_{f}\left(\frac{r}{2^{j \lambda}}\right)\right\}\right| \\
& \leq \sup \left\{\left|\frac{1}{32^{(j+1) \lambda}} m_{f}\left(\frac{r}{2^{(j+1) \lambda}}\right)-\frac{1}{32^{j \lambda}} m_{f}\left(\frac{r}{2^{j \lambda}}\right)\right|: 0 \leq j<k\right\} \\
& \leq \sup \left\{\left|\frac{1}{32}\right|^{j \lambda+\frac{\lambda-1}{2}} \Psi\left(\frac{r}{2^{j \lambda+\frac{\lambda+1}{2}}}, \frac{r}{2^{j \lambda+\frac{\lambda+1}{2}}}\right): 0 \leq j<k\right\} .
\end{aligned}
$$

By the application of (7) and allowing $k \rightarrow \infty$ in the above inequality implies that the validity of inequality (4). Employing (2), (3) and (7), for all $r_{1}, r_{2} \in S^{*}$, we have

$$
\begin{aligned}
\left|\Delta m_{f}\left(r_{1}, r_{2}\right)\right|= & \lim _{k \rightarrow \infty}\left|\frac{1}{32}\right|^{\lambda k}\left|\Delta m_{f}\left(\frac{r_{1}}{2^{\lambda k}}, \frac{r_{2}}{2^{\lambda k}}\right)\right| \\
& \leq \lim _{k \rightarrow \infty}\left|\frac{1}{32}\right|^{\lambda k} \Psi\left(\frac{r_{1}}{2^{\lambda k}}, \frac{r_{2}}{2^{\lambda k}}\right)=0
\end{aligned}
$$

which implies that (1) is satisfied by the mapping $M_{f}$ and which inturn shows that it is a reciprocal fifth power mapping. It is easy to verify the distinctness of $M_{f}$.

The outcomes of the following corollaries follow immediately from the results of Theorem 1 and so we provide only statements. These corollaries are pertinent to various other stability results of (1).

In the sequel, let $\eta \geq 0$ and $q \neq-5$, be fixed constants. Let $m_{f}: S^{*} \rightarrow T$ be a mapping.

Corollary 1. If the function $m_{f}$ satisfies

$$
\left|\Delta m_{f}\left(r_{1}, r_{2}\right)\right| \leq \eta
$$

for all $r_{1}, r_{2} \in S^{*}$, then a unique reciprocal fifth power mapping $M_{f}: S^{*} \rightarrow T$ exists and satisfying (1) and

$$
\left|m_{f}(r)-M_{f}(r)\right| \leq \eta
$$

for all $r \in S^{*}$.

Corollary 2. Suppose the mapping $m_{f}$ satisfies

$$
\left|\Delta m_{f}\left(r_{1}, r_{2}\right)\right| \leq \eta\left(\left|r_{1}\right|^{q}+\left|r_{2}\right|^{q}\right)
$$

for all $r_{1}, r_{2} \in S^{*}$, then a unique reciprocal fifth power mapping $M_{f}: S^{*} \rightarrow T$ satisfying (1) exists and

$$
\left|m_{f}(r)-M_{f}(r)\right| \leq \begin{cases}\frac{|2| \eta}{|2|^{q}}|r|^{q}, & q>-5 \\ |2| \eta|2|^{5}|r|^{q}, & q<-5\end{cases}
$$

for all $r \in S^{*}$.

Corollary 3. Suppose $a, b \in R$ with $q=a+b$ and the mapping $m_{f}$ satisfies

$$
\left|\Delta m_{f}\left(r_{1}, r_{2}\right)\right| \leq \eta\left|r_{1}\right|^{a}\left|r_{2}\right|^{b}
$$

for all $r_{1}, r_{2} \in S^{*}$. Then, there exists a unique reciprocal fifth power mapping $M_{f}: S^{*} \rightarrow T$ satisfying (1) and

$$
\left|m_{f}(r)-M_{f}(r)\right| \leq \begin{cases}\frac{\eta}{|2|^{q}}|r|^{q}, & q>-5 \\ \eta|2|^{5}|r|^{q}, & q<-5\end{cases}
$$

for all $r \in S^{*}$.

Corollary 4. Assume that the mapping $m_{f}$ satisfies

$$
\left|\Delta m_{f}\left(r_{1}, r_{2}\right)\right| \leq \eta\left(\left|r_{1}\right|^{\frac{q}{2}}\left|r_{2}\right|^{\frac{q}{2}}+\left(\left|r_{1}\right|^{q}+\left|r_{2}\right|^{q}\right)\right)
$$

for all $r_{1}, r_{2} \in S^{*}$. Then, a unique reciprocal fifth power mapping $M_{f}: S^{*} \rightarrow T$ exists and satisfies (1) and

$$
\left|m_{f}(r)-M_{f}(r)\right| \leq \begin{cases}\frac{|3| \eta}{|2|^{q}}|r|^{q}, & q>-5 \\ |3| \eta|2|^{5}|r|^{q}, & q<-5\end{cases}
$$

for all $r \in S^{*}$.

Inspired by the well-known the counter-example available in [4], we show that the stability result of equation (1) for the singular case $q=-5$ does not hold in Corollary 2 in the setting of non-zero real numbers. Let us assume the following function:

$$
\mu(r)= \begin{cases}\frac{\sigma}{r^{5}}, & \text { for } r \in(1, \infty) \\ \sigma, & \text { otherwise }\end{cases}
$$

where $\mu: R^{*} \rightarrow R$. Let $m_{f}: R^{*} \rightarrow R$ be defined by

$m_{f}(r)=\sum_{k=0}^{\infty} 32^{-k} \mu\left(2^{-k} r\right)$ 
for all $r \in R^{*}$.

Then the function $m_{f}$ becomes an apt example to prove that (1) is non-stable for $q=-5$ in Corollary 2 in the subsequent theorem.

Theorem 2. Suppose the function $m_{f}$ defined in (9) satisfies the ensuing inequality

$$
\left|\Delta m_{f}\left(r_{1}, r_{2}\right)\right| \leq \frac{33 \sigma}{8}\left(\left|r_{1}\right|^{-5}+\left|r_{2}\right|^{-5}\right)
$$

for all $r_{1}, r_{2} \in R^{*}$. Then a reciprocal fifth power mapping $M_{f}: R^{*} \rightarrow R$ and a constant $K>0$ do not exist such that

$$
\left|m_{f}(r)-M_{f}(r)\right| \leq K|r|^{-5}
$$

for all $r \in R^{*}$.

Proof. Firstly, let us show that $m_{f}$ satisfies (10). From the definition of $m_{f}$, we have

$$
\left|m_{f}(r)\right|=\left|\sum_{k=0}^{\infty} 32^{-k} \mu\left(2^{-k} r\right)\right| \leq \sum_{k=0}^{\infty} \frac{\beta}{32^{k}}=\frac{32}{31} \sigma,
$$

which concludes that $m_{f}$ has an upper bound $\frac{32}{31} \sigma$ on the set of real numbers. When $\left|r_{1}\right|^{-5}+\left|r_{2}\right|^{-5} \geq 1$, then $\left|\Delta m_{f}\left(r_{1}, r_{2}\right)\right|<\frac{33 \sigma}{8}$. On the other hand, suppose that $0<|u|^{-5}+|v|^{-5}<1$. Hence, we can find an integer $j>0$ such that

$$
\frac{1}{2^{j+1}} \leq\left|r_{1}\right|^{-5}+\left|r_{2}\right|^{-5}<\frac{1}{2^{j}} .
$$

Hence, the relation (12) produces $2^{j}\left(\left|r_{1}\right|^{-5}+\left|r_{2}\right|^{-5}\right)<1$, or equivalently: $2^{j} r_{1}^{-5}<1,2^{j} r_{2}^{-5}<1$. So, $\frac{r_{1}}{2^{j}}>1, \frac{r_{2}}{2^{j}}>1$. From the last inequalities, we observe that $\frac{r_{1}}{2^{j-1}}>2>1, \frac{r_{2}}{2^{j-1}}>2>1$ and as a consequence, we have

$$
\frac{1}{2^{j-1}}\left(r_{1}\right)>1, \frac{1}{2^{j-1}}\left(r_{2}\right)>1, \frac{1}{2^{j-1}}\left(r_{1}+r_{2}\right)>1 \text {. }
$$

Hence, for every $k=0,1,2, \ldots, j-1$, we obtain

$$
\frac{1}{2^{k}}\left(r_{1}\right)>1, \frac{1}{2^{k}}\left(r_{2}\right)>1, \frac{1}{2^{k}}\left(r_{1}+r_{2}\right)>1
$$

and $\Delta \mu\left(2^{-k} r_{1}, 2^{-k} r_{2}\right)=0$ for $k=0,1,2, \ldots, j-1$. Using

$$
\begin{aligned}
\left|\Delta m_{f}\left(r_{1}, r_{2}\right)\right| & \leq \sum_{k=j}^{\infty} \frac{\sigma}{2^{k}}+\sum_{k=j}^{\infty} \frac{\sigma}{32 \cdot 2^{k}} \\
& \leq \frac{33 \sigma}{32} \sum_{k=j}^{\infty} \frac{1}{2^{k}} \leq \frac{33 \sigma}{8}\left(\left|r_{1}\right|^{-5}+\left|r_{2}\right|^{-5}\right)
\end{aligned}
$$

for all $r_{1}, r_{2} \in R^{*}$. Hence, the inequality (10) holds. Now, we claim that (1) is not stable for $q=-5$ in Corollary 2. For this, let us consider a inverse fifth power mapping $m_{f}: R^{*} \rightarrow R$ exists and satisfies (11). Then, we have

$$
\left|m_{f}(r)\right| \leq(\mathrm{K}+1)|r|^{-5} \text {. }
$$

and the definition of $m_{f}$, we obtain

On the contrary, we opt an integer $m>0$ with $m \sigma>K+1$. If $r \in\left(1,2^{m-1}\right)$, then $2^{-k} r \in(1, \infty)$ for all $k=0,1,2, \ldots, m-1$ and hence we have

$$
\left|m_{f}(r)\right|=\sum_{k=0}^{\infty} \frac{\mu\left(2^{-k} r\right)}{32^{k}} \geq \sum_{k=0}^{m-1} \frac{32^{k} \sigma}{r^{5}}=\frac{m \sigma}{32^{k}}>(\mathrm{K}+1) r^{-5}
$$

which implies that (1) is non-stable for $q=-5$ in Corollary 2.

\section{VARIOUS NOTIONS ARISING IN OTHER FIELDS WITH THE SOLUTION OF (1)}

Many rational functions play dominant role in various fields such as physics, chemistry, biology, economics, medicine, mechanical engineering, cost analysis, etc. In this section, the solution of (1) is associated with different notions arising in physics, chemistry and mechanics.

1. Suppose a particle moves around a circular lane by the effect of an attractive central force, which is making the particle to move towards the centre point on the circle. Then we find this central force is proportional to the inverse fifth exponent of distance of the point from the point where the force is acting on the particle. Hence the central force is a function of reciprocal fifth power of the distance.

2. In the theory of wave spectrum, the rate of energy decay for the high frequency part is proportional to the inverse fifth exponent of frequency. In this case, the rate of energy decay is a function of reciprocal fifth power of frequency.

3. In kinetic theory of gases, molecules are conceived as portions of matter moving as single bodies, repelling by a force is proportional to the inverse fifth exponent of the distance between their centers of gravity. The inverse fifth power law is consistent with gas viscosity in terms of the time relaxation of stresses in the gas, a concept related to the macroscopic elastic properties of the gas.

4. Turbulent flow occurs in the large airways. The pressure drop in such turbulent flow is proportional to the inverse fifth exponent of radius of the airway.

\section{REFERENCES}

1. S. Alshybani, S. M. Vaezpour and R. Saadati, "Stability of the sextic functional equation in various spaces", J. Inequ. Special Func. vol. 9, no.4, pp. 8-27, 2018

2. A. Bodaghi and B. V. Senthil Kumar, "Estimation of inexact reciprocal-quintic and reciprocal-sextic functional equations", Mathematica, vol. 49, no. 82(1-2), pp. 3-14, 2017.

3. A. Ebadian, S. Zolfaghari, S. Ostadbashi and C. Park, "Approximation on the reciprocal functional equation in several variables in matrix non-Archimedean random normed spaces”, Adv. Diff. Equ. vol. 2015, no. 314,2015 .

4. Z. Gajda, "On the stability of additive mappings", Int. J. Math. Math Sci. vol. 14, pp. 431-434, 1991. 


\section{Approximations and Applications of a Reciprocal Fifth Power Mapping}

5. P. Gavruta, "A generalization of the Hyers-UlamRassias stability of approximately additive mappings", J. Math. Anal. Appl. vol. 184, pp. 431-436, 1994.

6. D. H. Hyers, "On the stability of the linear functional equation", Proc. Nat. Acad. Sci. U.S.A. vol. 27, pp. 222-224, 1941.

7. S. O. Kim, B. V. Senthil Kumar and A. Bodaghi, "Stability and non-stability of the reciprocal-cubic and reciprocal-quartic functional equations in non-Archimedean fields", Adv. Diff. Equ. vol. 77 pp. 1-12, 2017.

8. J. M. Rassias, "On approximately of approximately linear mappings by linear mappings", J. Funct. Anal. Vol. 46, pp. 126-130, 1982.

9. T. M. Rassias, "On the stability of the linear mapping in Banach spaces", Proc. Amer. Math. Soc. vol. 72, pp. 297-300, (1978).

10. K. Ravi, M. Arunkumar and J. M. Rassias, "On the Ulam stability for the orthogonally general Euler-Lagrange type functional equation", Int J. Math. Sci. vol. 3, no. 8, pp. 36-47, (2008).

11. K. Ravi and B. V. Senthil Kumar, "Ulam-Gavruta-Rassias stability of Rassias reciprocal functional equation", Global J. Appl. Math. Sci. vol. 3 , no. 1-2, pp. 57-79, (2010).

12. B. V. Senthil Kumar and H. Dutta, "Non-Archimedean stability of a generalized reciprocal-quadratic functional equation in several variables by direct and fixed point methods", Filomat, vol. 32, no. 9, pp. 3199-3209, 2018.

13. B.V. Senthil Kumar and H. Dutta, "Fuzzy stability of a rational functional equation and its relevance to system design", Int. J. General Syst. vol. 48, no.2, pp. 157-169, 2019.

14. B. V. Senthil Kumar and H. Dutta, "Approximation of multiplicative inverse undecic and duodecic functional equations", Math. Meth. Appl. Sci. vol. 42, pp. 1073-1081, 2019.

15. B. V. Senthil Kumar, H. Dutta and S. Sabarinathan, "Approximation of a system of rational functional equations of three variables", Int. J. Appl. Comput. Math. vol. 5, no. 3, pp. 1-16, 2019.

16. S. M. Ulam, "Problems in Modern Mathematics", Chapter VI, Wiley-Interscience, New York, 1964.

\section{AUTHORS PROFILE}

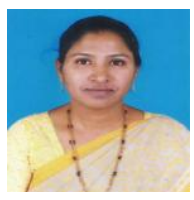

S. Merlin pursued her B.Sc Degree from University of Madras, in 1996. She got her M.Sc Degree in Madras Christian College, Tambaram in 1998 and her M.Phil Degree in Madras Christian College, Tambaram in 2000. She obtained her B.Ed. Degree from Cresent college of Education, Tiruvannamalai in 2010. She is having 19 years of teaching experience. At present she is working as an Assistant Professor in the Department of Mathematics, Kalasalingam Academy of Research and Education, Anand Nagar, Tamilnadu.

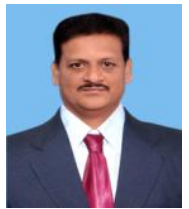

Dr. B. V. Senthil Kumar is serving in the Department of Information Technology, Nizwa College of Technology, Nizwa, Oman. His areas of interest are solution and stability of Functional, Differential and Difference equations, Operations Research, Statistics, and Discrete Mathematical Structures. He obtained his Ph. D. Degree in 2015. He has 19+ years of Teaching and 10 years of Research experience. He has published more than 50 research papers in reputed National and International Journals. He has co-authored 4 books on different titles published by World Scientific Publishing Company and Taylor \& Francis Group. He has contributed three chapters on various topics in textbooks published by John Wiley \& Sons and Springer. He has delivered invited talks in various institutions and also organized many academic and non-academic events. He is a member of many mathematical societies and a member of editorial committee for several journals. He is a reviewer of many international journals. 